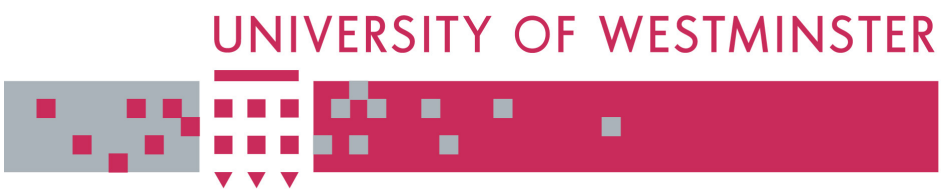

\title{
WestminsterResearch
}

http://www.wmin.ac.uk/westminsterresearch

\section{In vitro determination of skin bilirubin using chromatic modulation.}

\section{Tomtsis ${ }^{1}$ Vassilis Kodogiannis ${ }^{2}$ \\ D. Zissopoulos ${ }^{2}$}

${ }^{1}$ Macedonia Research Centre, TEI of West Macedonia, Kozani, Greece

${ }^{2}$ Harrow School of Computer Science, University of Westminster

Copyright (C [2001] IEEE. Reprinted from Building new bridges at the frontiers of engineering and medicine: 2001 Conference Proceedings of the 23rd Annual International Conference of the IEEE Engineering in Medicine and Biology Society. 25-28 October 2001, Istanbul, Turkey. pp. 3946-3949.

This material is posted here with permission of the IEEE. Such permission of the IEEE does not in any way imply IEEE endorsement of any of the University of Westminster's products or services. Internal or personal use of this material is permitted. However, permission to reprint/republish this material for advertising or promotional purposes or for creating new collective works for resale or redistribution must be obtained from the IEEE by writing to pubs-permissions@ieee.org. By choosing to view this document, you agree to all provisions of the copyright laws protecting it.

The WestminsterResearch online digital archive at the University of Westminster aims to make the research output of the University available to a wider audience. Copyright and Moral Rights remain with the authors and/or copyright owners. Users are permitted to download and/or print one copy for non-commercial private study or research. Further distribution and any use of material from within this archive for profit-making enterprises or for commercial gain is strictly forbidden.

Whilst further distribution of specific materials from within this archive is forbidden, you may freely distribute the URL of WestminsterResearch. (http://www.wmin.ac.uk/westminsterresearch).

In case of abuse or copyright appearing without permission e-mail wattsn@wmin.ac.uk. 


\title{
IN VITRO DETERMINATION OF SKIN BILIRUBIN USING CHROMATIC MODULATION
}

\author{
D. Tomtsis ${ }^{1}$, V. Kodogiannis ${ }^{2}$, D. Zissopoulos ${ }^{1}$ \\ ${ }^{1}$ Macedonia Research Centre, TEI of West Macedonia, Kozani, GR-50100, Greece \\ ${ }^{2}$ Computer Science Dept, University of Westminster, London HA1 3TP, United Kingdom
}

\begin{abstract}
A clinical examination system is presented to analyse the yellowness presented on the skin of premature newborn babies, namely transcutaneous bilirubin (TcB). The system is based on chromatic modulation methods to extract, process and analyse data in order to determine the spectral chromaticity of a baby's tissue without taking blood samples. Chromatic bilirubin monitoring tests have shown a high correlation and a linear relationship between serum bilirubin (SB) and a chromatic parameter, namely Hue angle $(\mathrm{H})$. The chromatic TcB results are highly predictive of SB levels in neonatal babies. The proposed chromatic system can be used to make decisions about transfusions or phototherapy in neonates, hence acting as a screening device to determine when a laboratory measurement of $\mathrm{SB}$ is needed.
\end{abstract}

Keywords - chromatic modulation, chromaticity, remote monitoring, skin bilirubin, serum bilirubin, newborn babies.

\section{INTRODUCTION}

Prematurely born, or neonatal babies, due to their early separation from mother's physiological support systems are unable to maintain several control mechanisms in a safe state. Such mechanisms include the assured delivery of appropriate levels of oxygen to all cells in the body and cleansing of their blood while the liver adjusts to its proper function [1]. A common disorder in newborns related to blood and liver diseases is Jaundice that is characterised by deep yellowness of the skin. Jaundice is caused by the excess production or inadequate excretion of bilirubin, a yellow bile pigment formed from haemoglobin breakdown products after decomposition of red blood cells [2]. Bilirubin is present in neonates mainly in two ways; either in the form of accumulation in the fat just below the skin, or in a more toxic form in the blood plasma. The former is usually treated by phototherapy that isomerises un-conjugated bilirubin into water-soluble derivatives and facilitates their excretion from the body, while the latter can cause severe brain damage.

Bilirubin measurement is one of the most frequently performed tests on critically ill newborn babies in intensive care units. The general procedure for examining a neonate for bilirubin involves two steps: Initially, a visual examination of the baby is been performed while in a second step, this procedure is followed by blood sampling and laboratory analysis to provide objective and accurate measures of SB levels [3]. While blood sampling causes usually discomfort and pain to the neonate and is associated with the risk of infection especially when it is performed frequently, laboratory analysis is a complex and time consuming procedure [4].

A number of research groups have attempted to develop non-invasive systems that could measure skin bilirubin levels without the aid of blood samples. Gosset [5] described the use of an icterometer as a screening device for gauging the degree of jaundice in newborns. Ballowitz et al. discussed the possibility to quantify the degree of bilirubin using measurements of the spectral reflectance of the tissue in the wavelength range 430 to $520 \mathrm{~nm}$ [6]. Also, a non-invasive prototype device was used to measure the concentration of bilirubin by analysing the spectral reflectance of a neonate's tissue and correlation factors between these two quantities have been discussed [7]. The correlation coefficient between bilirubin concentration and measurements of spectral reflectance from the tissue was reported to be 0.94 at five wavelengths in the wavelength range 420 to $630 \mathrm{~nm}$ [8]. However, results obtained by such methods are lacking in accuracy and practical efficiency.

In this study, a non-invasive monitoring system is presented to determine the chromaticity of a baby's tissue by optical methods and chromatic techniques, which are based on wide-band rather than narrow-band detection techniques. Emphasis has been given in the design of the system in order to meet clinical requirements such as required accuracy, and friendly usage.

\section{CHROMATIC MODULATION THEORY}

The essence of chromatic modulation - an integrated form of spectral monitoring - is the utilisation of polychromatic light for sensing spectral changes by monitoring the total profile of an optical signal within a spectral power distribution. Chromatic changes can be monitored by a number (n) of detectors with overlapping spectral responses. The output of each detector may then be expressed as [9]

$$
\mathrm{V}_{\mathrm{n}}=\int \mathrm{P}(\lambda) \mathrm{R}_{\mathrm{n}}(\lambda) \mathrm{d} \lambda
$$

where $P(\lambda)$ is the spectral power distribution in the optical signal and $R_{n}(\lambda)$ is the wavelength responsivity of the $n^{\text {th }}$ detector and $\lambda$ is the wavelength. The colour model representing this mathematical formalism is generally called RGB and it is widely used in self-luminous display technologies.

Each detector output may also be intensity normalised according to: 


$$
u_{n}=\frac{V_{n}}{\sum_{n} V_{T}}
$$

and chromaticity maps may be formed in terms of the coordinates $u_{1}, u_{2}, \ldots u_{(n-1)}$. The special case of $n=3$ leads to a two-dimensional chromaticity map $\left(\mathrm{u}_{1}: \mathrm{u}_{2}\right)$ on which changes in optical signals may be traced. The special case when $R_{1}(\lambda), R_{2}(\lambda), R_{3}(\lambda)$ correspond to the responsivities of the human eye leads to the chromaticity map reducing to the CIE diagram of colour science [10]. The colour model representing this mathematical formalism is called $\mathrm{L}_{\mathrm{XY}}$ and provides the relative magnitudes of the tri-stimulus values (i.e. $x=u_{1} ; y=u_{2} ; z=u_{3}$ ). However, this method of displaying chromatic information does not easily lead to the identification of signal changes in terms of fundamental signal properties. An alternative approach to the processing of the signals from such chromatic detectors overcomes this limitation. For the tri-stimulus case $(n=3)$ this approach utilises three chromatic parameters, namely dominant wavelength $(\mathrm{H})$, intensity $(\mathrm{L})$ and degree of monochromaticity or spectral width (S) which are defined as follows:

$$
H=120\left[m_{i}+\left(\frac{V_{i}-V_{\min }}{V_{i}+V_{j}-2 V_{\min }}\right)\right]
$$

with $m_{\mathrm{i}}=0$ for $\mathrm{i}=1, \mathrm{j}=2, \mathrm{~V}_{\min }=\mathrm{V}_{3}$,

$$
\mathrm{m}_{\mathrm{i}}=1 \text { for } \mathrm{i}=2, \mathrm{j}=3, \mathrm{~V}_{\min }=\mathrm{V}_{1} \text {, }
$$$$
\mathrm{m}_{\mathrm{i}}=2 \text { for } \mathrm{i}=3, \mathrm{j}=1, \mathrm{~V}_{\min }=\mathrm{V}_{2}
$$$$
L=100\left[\frac{V_{\max }+V_{\min }}{2}\right]
$$

$$
S=100\left[\frac{V_{\max }-V_{\min }}{200 m_{2}-m_{3}\left(V_{\max }+V_{\min }\right)}\right]
$$

with $m_{2}=0, m_{3}=-1$ for $L \leq 50$ and $m_{2}=m_{3}=1$ for $L>50 . V_{\min }$ and $\mathrm{V}_{\max }$ are the minimum and maximum detector outputs [10].

\section{THE EXPERIMENTAL SYSTEM}

The proposed remote monitoring system is consisted of three parts: a colour CCD camera recorder, a colour frame grabber card (C.F.G.), and a host personal computer. Fig. 1 illustrates graphically the configuration of the system. The camera converts the spatially distributed optical information into analogue electric signals, which are accepted by the colour frame grabber card (Hauppauge's WinTV Cinema). The signal is then digitised and stored in the card's frame memory. A portable JVC GR-AX200 high-resolution camcorder was used, which was able, to record the acquired information frame by frame on a VCR tape (off-line operation), or to redirect it to the colour frame grabber card (real time operation). The proposed experimental apparatus is characterised by its portability as only the camera can be used during the data acquisition stage and then in a off-line process to be interfaced with the rest of the system for further data processing and analysis.

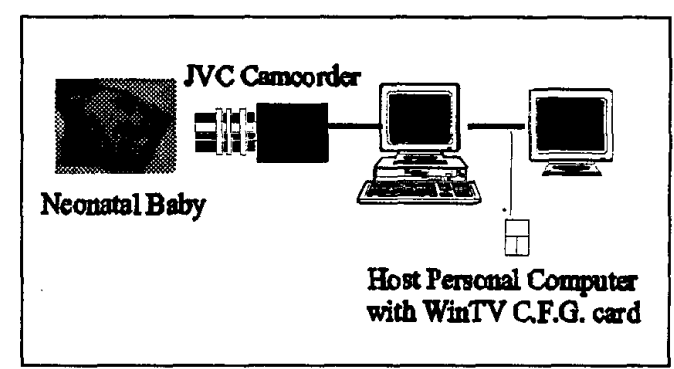

Fig. 1. Illustration of the proposed remote monitoring system

During the clinical operation of the proposed chromaticbased system an external light source was used to assist the event being monitored. Thus, special care was taken to compensate for any spectral variation due to this light source. As a result, each recorded image contained a colour chart, which was used for colour calibration, as shown in Fig. 2.

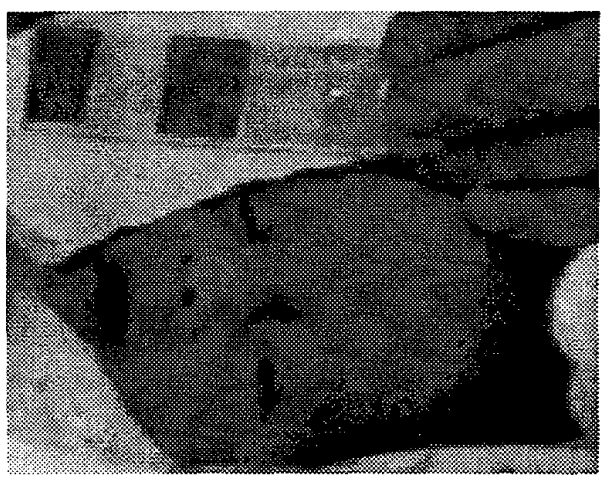

Fig. 2: Proposed colour chart

As shown in the same Fig., a transparent plastic slide was applied to the neonate's nose to remove haemoglobin. The clinical remote monitoring system was then utilised to monitor the spectral chromaticities of the tissue of a number of ill, prematurely born babies, in order to chromatically analyse the data and predict the bilinubin level of each neonate.

\section{EXPERIMENTAL METHOD}

\section{A. Overview}

The clinical remote monitoring system accepted a complete spectrum of visible light from the baby's tissue, which was either processed immediately if the system was used on-line or stored as an image in the colour frame grabber card to be processed at a later stage if the system was used off-line. Computer software obtained the RGB chromatic values from the neonates nose, performed colour calibration (to remove any errors introduced by non-uniform illumination or light fluctuations in the clinical environment), and converted these readings to a number of different chromatic systems for 
easier analysis. For good repeatability and noise reduction an $8 \times 8$ local averaging mask was used to obtain the average RGB chromatic parameters. By comparing the $\mathrm{TcB}$ data in a chromatic format from the baby against reference data from the colour chart in the same format stored in the host personal computer, the remote monitoring system was able to predict the SB level of a baby even when none was visible to the naked eye. When the remote monitoring system was used, a chromatic reading from the light reflected from the baby's tissue was produced which correlated to a physical parameter such as a laboratory value for SB level. Measurements have been made on 15 different neonates each having different levels of bilirubin in their blood as determined by blood analysis. The measurements were taken with the CCD camera viewing the neonate's nose tissue. The later was pressed onto a transparent plastic slide in order to remove haemoglobin from the tissue prior to the chromatic monitoring.

\section{B. Chromatic Calibration}

The chromatic information received by the remote monitoring system depends on the lighting conditions of the surrounding environment. In other words, the chromatic coordinates of an object under daylight conditions will be different than those under artificial light conditions. In. the later case, alternative procedures are usually adopted and incorporated into the colour models of section II to allow for lightness adaptation. In this paper a modified Von Kries transformation [11] has been developed with the use of any carefully selected colour as a reference one. In this procedure if a stimulus gives rise to the $r, g$, and $b$ colour responses then the resulting responses will depend on $r / r_{c}, g / g_{c}, b / b_{c}$, where $r_{c}, g_{c}$, and $b_{c}$ are the chromatic co-ordinates of the reference colour. For a stimulus in the state considered to have the same colour appearance as the same stimulus in a reference state it is necessary that

$$
\frac{r}{r_{c}}=\frac{r^{l}}{r_{c}^{l}}, \frac{g}{g_{c}}=\frac{g}{g_{c}^{l}}, \frac{b}{b_{c}}=\frac{b^{l}}{b_{c}^{l}}
$$

where $r_{c}, g_{c}^{l}$ and $b_{c}^{\prime}$ are the colour responses of the reference colour in the reference state. Hence

$$
r^{\prime}=\left(\frac{r_{c}^{\prime}}{r_{c}}\right) r, g^{\prime}=\left(\frac{g_{c}^{\prime}}{g_{c}}\right) g, b^{\prime}=\left(\frac{b_{c}}{b_{c}}\right) b
$$

The resulting adapted variables $r, g, b$, obtained from (7), can then be used to calculate the $L_{X Y}$ tri-stimulus values as well as their HLS representation. The developed calibration method for correcting the variations in RGB chromatic values focus on removing the RGB errors introduced by nonuniform illumination and external light fluctuations of the clinical environment. The algorithm for chromatic calibration is based upon the use of a custom colour chart and developed as a pre-processing tool for colour image analysis. The colour chart consisted of six different colour patches, each one at a different wavelength of the visible spectrum, as shown in Fig. 2. Each colour patch in the chart was about 5 $\mathrm{cm}$ square. The chromatic values of each patch were recorded under controlled laboratory lighting conditions, which have been used as reference information for any further processing.

\section{RESULTS}

The experimental method was used to produce TcB measurements in a number of different chromatic systems. Fig. 3 shows the measured chromatic variation in $L_{X Y}$ space as a function of actual SB levels for the 15 neonates participating in the tests.

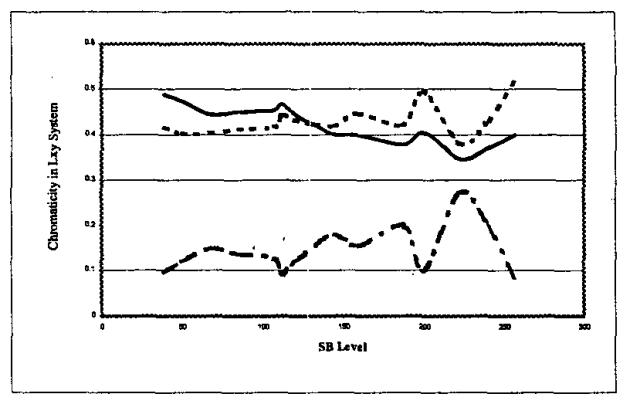

Fig. 3: $\mathrm{L}_{X Y}$ variation (chromaticities $X, Y, Z$ ) against $S B$ level. Straight line: chrom. X, dotted line: chrom. Y, dashed line: chrom. Z

There is considerable variation of each measured quantity, but identification of important characteristics or trends from these traces is obviously difficult since there is no indication about the expected lack of redness as the SB level is increased.

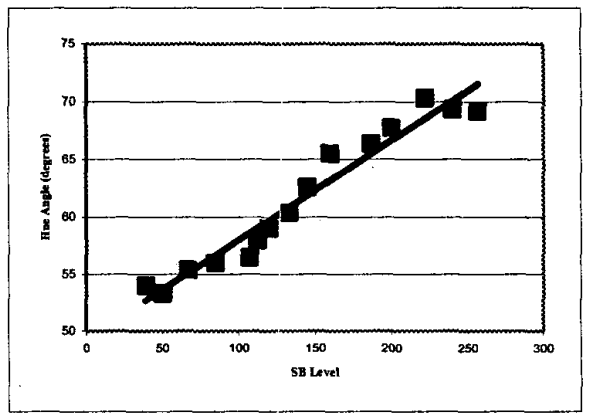

Fig. 4: Linear regression between Hue angle and SB level. Square: Hue angle, full line: linear regression.

A simple transformation of the same data to the HLS chromatic space reveals that there is a clear trend between bilirubin concentration and hue angle (Fig. 4), which represents a variation from 53 to 73 degrees approximately (a shift from the red towards the yellow side of the spectrum). The modulation depth is 20 degrees, a significant dominant wavelength variation from higher to lower wavelengths. 
Linear correlation was used to quantify the degree of such correlation between the measured quantities in $\mathrm{L}_{X Y}$ and HLS systems and SB levels. Table I summarises the correlation coefficients obtained when SB levels were correlated with TcB readings. As it is seen, the best correlation factors were obtained for hue angle and chromaticity $X$. The least square fit method was then applied to the quantity with the highest correlation factor (bilirubin and hue angle) to produce the best line between the experimental data.

TABLE I

Linear correlation between chromatic quantities and SB level

\begin{tabular}{|l|l|}
\hline Correlated Quantities & Correlation Coefficient \\
\hline SB Level and Hue Angle & 0.975 \\
\hline SB Level and Saturation & -0.344 \\
\hline SB Level and Lightness & -0.377 \\
\hline SB Level and Chromaticity X & -0.884 \\
\hline SB Level and Chromaticity Y & 0.496 \\
\hline SB Level and Chromaticity $Z$ & 0.387 \\
\hline
\end{tabular}

This type of linear regression produced a linear equation, which can be used to calibrate the system:

$$
B=-571.87+11.59 * H
$$

where $B$ denotes SB level and $H$ represents Hue Angle in degrees. The experimental data points for hue angle together with the best line fit results are plotted and presented in Fig. 4. The good correlation between bilinubin concentration in the skin and that in the blood by SB determination methods shows that there is generally a uniform, balanced distribution between the bilirubin within the blood vessels and that outside. Hence, the chromatic TcB measurement technique may be used as an alternative method to those based on blood sampling to produce the same results. Both chromatic quantities, hue angle and chromaticity $X$, present a high correlation coefficient with SB level, but as expected, hue angle demonstrates the best correlation because it shows the dominant wavelength of the neonates skin rather than the percentage of the red colour which is presented by chromaticity $X$. In general terms, the graphs show how a simple transformation of the data from the $L_{X Y}$ colour space to the HLS form can simplify the identification of trends between experimental (TcB readings) and actual results ( $\mathrm{SB}$ level).

\section{CONCLUSION}

Serum bilirubin (SB) has been the preferred method of detecting hyperbilirubinemia in newborns. Blood sampling exposes the neonate to trauma and risk of infection. A noninvasive system for predicting SB levels in newborns diminishes the need to take blood samples. Such a clinical examination system has been developed to predict the SB levels of premature newborn babies. The system has been based on chromatic modulation methods to extract, process and analyse data in order to determine the chromaticity of a baby's tissue without taking blood samples. The noninvasive clinical system can offer a rapid assessment of bilirubin concentration, with performance parallel to measurements obtained by blood testing, by transforming a complex and time consuming process, such as existing clinical laboratory methods, into a simple procedure. The continuous monitoring of Hue angle would also allow a physician to follow trends in the neonate's condition. On the other hand, off-line operation is also possible when a quick return of a measurement is not required which enhances the operational flexibility of the clinical system. The system can be further improved to perform automatic and unsupervised data extraction by object tracking algorithms, which can be used to continuously locate in real time the position of the colour chart needed for colour calibration and the area on the tissue to be analysed.

\section{REFERENCES}

[1] C.E. Ahlfors, "Criteria for exchange transfusion in jaundiced newborns," Paediatrics, vol. 94, pp 484-494, 1994.

[2] S.B. Turkel, "Autopsy findings associated with neonatal hyperbilirubinemia," Clin Perinatol, vol. 17, pp. 381-396, 1990.

[3] J. Dai, D.M. Parry, J. Krahn, "Transcutaneous bilirubinometry: Its role in the assessment of neonatal jaundice," Clinical Biochemistry, vol. 30, pp. 1-9, 1997.

[4] H. Varley, Practical Clinical Biochemistry, Medical Books Ltd., 4th edition, 1978.

[5] I.H. Gosset, "A Perspex icterometer for neonates," Lancet vol. 1, pp. 87-88, 1960.

[6] L. Ballowitz, M.E. Avery, "Spectral Reflectance of the Skin: Studies on Infant and Adult Humans," Biology of the Neonate, vol .15, pp. 348-360, 1970.

[7] R.E. Hannemann, D.P. DeWitt, J.F. Wiechel, "Neonatal Serum Bilirubin from Skin Reflectance," Paediatric Research, vol. 12, pp. 207-210, 1978.

[8] K.J. Peeve, L. Mumford, R. Bruce, S.J. Gross, "Estimation of Serum Bilirubin by Spectral Reflectance of the Skin," Paediatric Research, vol. 12, pp 532, 1978.

[9] H. Levkowitz, G.T. Herman, "GLHS: A Generalised lightness, hue, and saturation colour model," Graphical models and image processing, vol. 55, no. 4, pp. 271-285, 1993.

[10] J.D. Foley, A.V. Dam, S.K. Feiner, J.F. Hughes JF, Computer graphics: Principles and practice, 1995, Addison-Wesley.

[11] R.W. Hunt, Measuring Colour, J. Wiley and Sons, New York, 1987. 